\title{
Single wave extraction in continuous intracranial pressure signal with lifting wavelet transformation and discrimination rules
}

\author{
Zhong Ji, Lan Zhu, Xing Yang and Lipeng Jiang
}

\begin{abstract}
Objective: This article describes a novel method for processing continuous intracranial pressure (ICP) signals with lifting wavelet transformation and discrimination rules for ICP waveform morphology.

Methods: First, lifting wavelet was applied to detect the extreme points of ICP waveform preliminarily; then, the extreme points that were undetected or falsely detected are determined by using the discrimination rules repeatedly; finally, those falsely detected and undetected points were removed or corrected to improve the accuracy of identified individual pulse.
\end{abstract}

Results: The algorithm was employed to analyze continuous ICP signals of nine patients. Signals were recorded for 30 min each. Each signal was divided into 30-s segments and analyzed. The accuracy rate of $98.58 \%$ was obtained.

Conclusion: The method described in this article has given a possibility for the clinical use of ICP waveform. By identifying the single ICP wave effectively, not only mean ICP but also single ICP wave amplitude and latency can be computed precisely with this new method.

Keywords: intracranial pressure, single wave extraction, lifting wavelet, discrimination rules

\section{Introduction}

Mean intracranial pressure (ICP) is often regarded as a clinical indicator during continuous ICP monitoring, and is computed according to the sum of pressure levels divided by number of samples. However, the ICP wave parameters of a single ICP wave, such as ICP wave amplitude and latency, can provide the information that is not given in mean ICP [1-3]. Many studies have indicated that the ICP wave parameters are related to intracranial pressure-volume compensatory reserve capacity. $\mathrm{Hu}$ et al. [4] also pointed out that ICP elevation could be predicted by the prescient change of ICP waveform morphology. The present research situation of single ICP wave identification and its importance in clinical practice has been discussed in other articles very well, and there are several methods developed to analyze the continuous ICP signals [1-7]. We have developed an

\footnotetext{
* Correspondence: jizhong@cqu.edu.cn Key Laboratory of Biorheological Science and Technology of Ministry of Education, Bioengineering College of Chongqing University, Chongqing 400030, China
}

alternative single wave identification method that combined lifting wavelet transform with waveform discrimination rules. In the premise of not reducing the accuracy of single wave identification, the method decreased the single wave parameters that required identification, and simplified the identification process.

Since the continuous ICP signal is dynamic and often interfered by noise, the feature points used to identify the single ICP wave may be inconspicuous. Wavelet transform is a signal processing method broadly used for signal de-noising and feature extraction $[8,9]$. However, in practice, because of the variation of wavelet bases, it is often needed to try different wavelet bases to find a suitable one according to the wave features of analyzed signal. The research and discussion for the first generation of wavelet are conducted within the framework of Fourier analysis, i.e., the problem is analyzed in the view of frequency domain. Sweldens used a new wavelet construction algorithm that does not rely on Fourier transformation, but on lifting scheme to construct wavelet in time domain, then he established the 
second generation wavelet transform theory $[10,11]$. Compared with the first generation wavelet, the lifting wavelet can be used to self-define wavelet construction based on the characteristics of the analyzed signal. It contributes to a better real-time performance of the diagnosis system by reducing calculation. Based on the lifting scheme, according to the characteristics of a single ICP wave, an appropriate wavelet can be constructed to remove noise effectively; furthermore, the discrimination rules can be developed for the extraction of single ICP waves. In this way, the extreme points of single ICP waves can be detected with higher accuracy.

\section{Methods}

\subsection{Wavelet transform based on lifting scheme}

The wavelet decomposition based on lifting scheme could be divided into the following three stages: split, prediction, and update $[10,11]$.

(1) Split

First, the input signal $s_{i}$ was divided into two smaller subsets $s_{i-1}^{\prime}$ and $d_{i-1}^{\prime}$, where $d_{i-1}^{\prime}$ was also known as wavelet subset. The simplest split was that $s_{i}$ was divided into two groups according to parity, then $s_{i-1}^{\prime}$ was known as the even sequence and $d_{i-1}^{\prime}$ known as the odd sequence. This split wavelet was called the Lazy Wavelet, which could be expressed as

$$
\text { split }\left(s_{i}\right)=\left(s_{i-1}^{\prime}, d^{\prime}{ }_{i-1}\right) .
$$

\section{(2) Prediction}

Based on the correlation of raw data, the predicted $P\left(s_{i-}{ }^{\prime}\right.$ 1) of the even sequence $s_{i-1}^{\prime}$ was used to predict (or interpolate) the odd sequence $d_{i-1}^{\prime}$. In practice, even though it was impossible to predict the subset $d_{i-1}^{\prime}$ accurately, it was possible to make $P\left(s_{i-1}^{\prime}\right)$ very close to $d_{i-1}^{\prime}$, so $P\left(s_{i-1}^{\prime}\right)$ could be used to replace the original $d_{i-1}^{\prime}$ with the difference between $d_{i-1}^{\prime}$ and $P\left(s_{i-1}^{\prime}\right)$, then the generated $d_{i-1}$ would contain less information than the original $d_{i-1}^{\prime}$, that is

$$
d_{i-1}=d_{i-1}^{\prime}-P\left(s_{i-1}^{\prime}\right) .
$$

\section{(3) Update}

The idea of update was to find a better subset $s_{i-1}$, which maintained some scalar features $Q\left(s_{i}\right)$ (such as invariant mean and vanishing moment) of the original signal, i.e., $Q\left(s_{i-1}\right)=Q\left(s_{i}\right)$. The computed wavelet subset $d_{i-1}$ could be used to update $s_{i-1}^{\prime}$, which made the latter maintain the same scalar features. An operator $U$ could be constructed to update $s_{i-1}$, which was defined as follows:

$$
s_{i-1}=s_{i-1}^{\prime}+U\left(d_{i-1}\right),
$$

where the obtained subset $s_{i-1}$ was smaller than the original signal set $s_{i}$, and the wavelet subset $d_{i-1}$ could also be obtained, i.e., the signal had been implemented wavelet transformation.

Among the three stages, the prediction and the updating steps were the core of wavelet lifting. The high-frequency and suitable low-frequency information could be acquired, respectively, by predicting and updating steps. It is easy to understand that the above algorithm only needs the output of former updating step, so that the former data stream of each point could be replaced by the new one. Namely bit operation, which did not occupy the system memory, could be achieved.

It is easily to obtain the inverse transformation of the lifting scheme from its positive transformation, only by changing the direction, as well as plus and minus sign of the data stream. Namely, the reconstruction was composed of restoring update, prediction, and the decomposed subset combination, that is

$$
\begin{aligned}
& s_{i-1}^{\prime}=s_{i-1}-U\left(d_{i-1}\right), \\
& d_{i-1}^{\prime}=d_{i-1}+P\left(s_{i-1}^{\prime}\right), \\
& \left(s_{i-1}^{\prime} d_{i-1}^{\prime}\right)=\operatorname{merge}\left(s_{i}\right) .
\end{aligned}
$$

In the algorithm, $P$ and $U$ could be chosen to construct the wavelet and scaling functions with some characteristics. The split and merging process of a single lifting are shown in Figure 1.

\subsection{Definitions of single ICP wave parameters}

The definitions of parameters were introduced to describe the characteristics of a single ICP wave [1]. In Figure 2, the starting minimum diastolic pressure of the single wave $(P \min 1)$ defines its start, the ending minimum diastolic pressure $(P \min 2)$ defines the end, and the maximum systolic pressure $(P \max 1)$ defines the maximum of the single wave. In this article, the time duration $\mathrm{d} W 2$ of two maximum systolic pressures of adjacent ICP waves, a new parameter, was also introduced to discriminate whether the detected extreme points were correct. The single ICP wave amplitude $\mathrm{d} P$ was defined as the pressure difference between $P \min 1$ and $P \max 1$, the latency of a single ICP wave was the time interval when the pressure changed from $P \min 1$ to $P \max$, the $\mathrm{d} W 1$ defined the time duration of a single ICP wave between $P$ min 1 and Pmin2, and the dW2 defined the time duration between the two peaks $P \max 1$ and Pmax 2 of two adjacent ICP waves.

Based on the definitions, the single ICP wave parameters could be computed with the following formulas after extracting the single ICP wave with the lifting wavelet algorithm shown in Section 4:

$$
\mathrm{d} P=P \max 1 . y-P \min 1 . y
$$




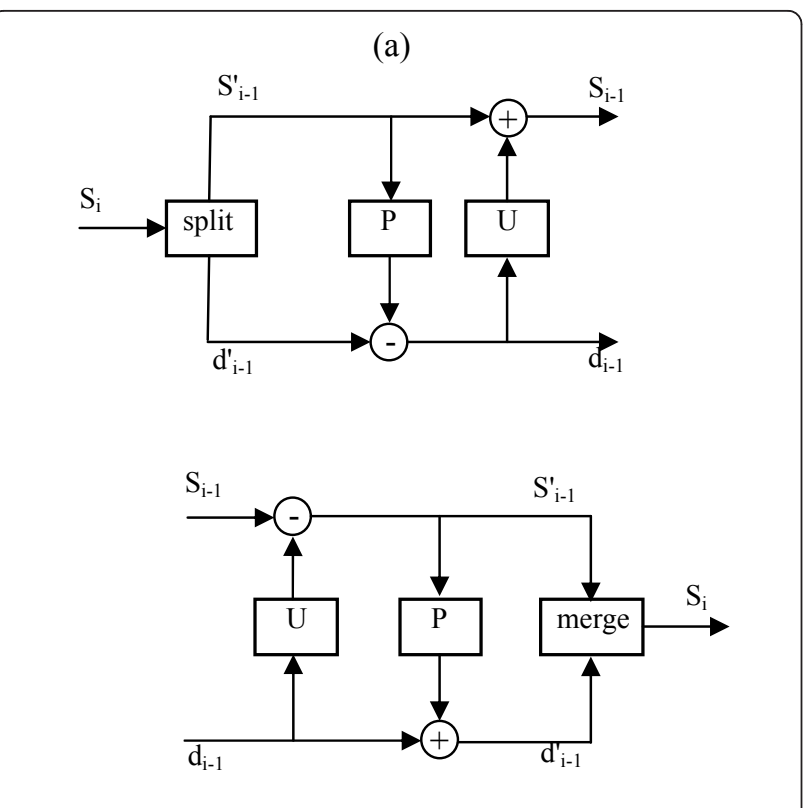

(b)

Figure 1 Splitting and merging process of once lifting

$$
\begin{aligned}
& \mathrm{d} T=P \max 1 . x-P \min 1 . x \\
& \mathrm{~d} W 1=P \min 2 . x-P \min 1 . x \\
& \mathrm{~d} W 2=P \max 2 . x-P \max 1 . x
\end{aligned}
$$

where $x$ was the time value of the feature point and.$y$ was the pressure value.

\subsection{Algorithm process for single ICP wave extraction}

According to the feature of a single ICP wave, the peak and valley of a single ICP wave were regarded as singular points in continuous ICP wave, so we developed a new algorithm to extract single ICP wave based on lifting wavelet and discrimination rules as follows:

(1) Preprocess the sampled ICP signals and segment them by $N$ section per minute, where $2 \leq N \leq 10$;

(2) Split every segment of ICP wave into LEVEL layers with lifting wavelet. Thereby, the detail signal $D=\left\{d_{i}\right\}$ and approximate signal $S=\left\{s_{i}\right\}, i=1,2, \ldots$, LEVEL of every layer was obtained. Then, the detail parts were summed up to get the total odd sequence (detail signals) which was transformed from original signal with lifting wavelet;

(3) Construct sliding window to further process the odd sequence. The width of sliding window was $w=f_{\mathrm{s}} /$ $2 f$, and the sliding coefficient was $\delta=f_{\mathrm{s}} / 2 f$, which was determined by the sampling frequency $f_{\mathrm{s}}$ and the cardiac beat period $f$;
(4) Calculate module mini-max values of ICP signal in every sliding window as the feature points of the single ICP waves. These computed positive and negative module maximums were regarded as the peaks and valleys.

Lifting wavelet transformation was applied to the above four steps to get the mini-max points. However, if only lifting wavelet transformation was used to identify the single ICP wave, some extreme points might be undetected or detected falsely. Further analysis indicated that after the above four steps, several abnormal cases of extreme points existed, which were identified with frames in Table 1. These abnormal cases included: (a) two minimum points close to each other were identified between two maximum points; (b) two maximum points close to each other were identified between two minimum points; (c) no maximum point was identified between two minimum points; and (d) no minimum point was identified between two maximum points.

Based on the above-mentioned definitions in Figure 2 and formulas (7) to (10), $\mathrm{d} W 1, \mathrm{~d} W 2, \mathrm{~d} T$, and $\mathrm{d} P$ were calculated, and the ranges of the first three parameters were determined: $500 \mathrm{~ms}<\mathrm{d} W 1<1200 \mathrm{~ms}, 500 \mathrm{~ms}<$ $\mathrm{d} W 2<1200 \mathrm{~ms}, 100 \mathrm{~ms}<\mathrm{d} T<250 \mathrm{~ms}$; and single ICP wave amplitude $(\mathrm{d} P)$ should be between 3 and 20.0 $\mathrm{mmHg}$. Further discrimination processing was made to find out the missing extreme points and filter the false ones, therefore the identification ability of ICP waveform was improved.

Specific discrimination rules were as follows:

(5) Arrange the mini-max points acquired by steps (1) to (4) in chronological order, and then calculate waveform parameters $\mathrm{d} T, \mathrm{~d} W 1, \mathrm{~d} W 2$, and $\mathrm{d} P$;

(6) Discriminate whether extreme points are missing or detected false according to the ranges of above-mentioned parameters. The followings were specific ways for discrimination:

(a) When dW1 was within the normal range, if two maximum points were identified between two minimum points and this $\mathrm{d} W 2$ was below the lower limit, then a maximum point was detected false. Comparing the amplitudes of these two maximum points, and the bigger one was chosen as the final maximum point, under the premise that $\mathrm{d} P$ was within the normal range;

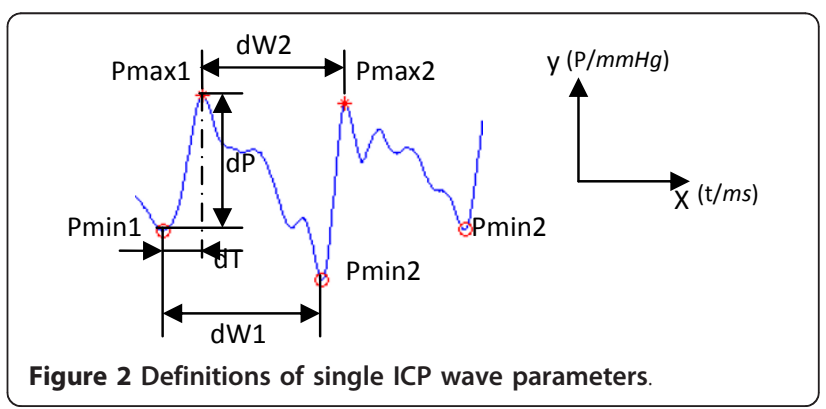


Table 1 Relative sampling positions of maximum and minimum points of first 30-s signal segment, the boxed values denoted the false detected points, or there were undetected points between the two adjacent boxed values

\begin{tabular}{|c|c|c|c|c|c|c|c|c|}
\hline \multirow{2}{*}{$\frac{P \max }{P \operatorname{Pmin}}$} & \multicolumn{3}{|c|}{192} & \multirow[t]{2}{*}{471} & \multicolumn{3}{|c|}{733} & \multirow[t]{2}{*}{1016} \\
\hline & 140 & & 383 & & 686 & & 947 & \\
\hline Pmax & & 1272 & & 1542 & & 1819 & 1821 & \\
\hline Pmin & 1231 & & 1486 & & 1749 & & & 2029 \\
\hline Pmax & 2081 & & 2367 & & 2619 & & 2923 & \\
\hline Pmin & & 2288 & & 2572 & & 2829 & & 3097 \\
\hline Pmax & 3160 & & & 3416 & & 3707 & & 3963 \\
\hline Pmin & & 3374 & 3381 & & 3661 & & 3920 & \\
\hline Pmax & & 4241 & 4505 & & & 4761 & & 5053 \\
\hline Pmin & 4174 & & & 4663 & 4720 & & 4977 & \\
\hline Pmax & & 5304 & & 5561 & & 5865 & & 6107 \\
\hline Pmin & 5251 & & 5517 & & 5777 & & 6056 & \\
\hline Pmax & & 6415 & & 6650 & & 6907 & & 7195 \\
\hline Pmin & 6320 & & 6574 & & 6862 & & 7123 & \\
\hline Pmax & 7285 & & & 7736 & & 8024 & 8076 & \\
\hline Pmin & & 7406 & 7692 & & 7960 & & & 8239 \\
\hline Pmax & & 8570 & 8581 & & 8856 & & 9145 & \\
\hline Pmin & 8526 & & & 8811 & & 9071 & & 9359 \\
\hline Pmax & & 9403 & & 9688 & & 9982 & 10258 & \\
\hline Pmin & 9361 & & 9641 & & 9907 & & & 10293 \\
\hline Pmax & & 10521 & & 10819 & & 11064 & & 11354 \\
\hline Pmin & 10467 & & 10738 & & 11024 & & 11286 & \\
\hline Pmax & & 11612 & & 11904 & & & & \\
\hline Pmin & 11565 & & 11825 & & & & & \\
\hline
\end{tabular}

(b) When $\mathrm{d} W 1$ was within the normal range, if no maximum point was identified between two minimum points and $\mathrm{d} W 2$ was beyond the normal range, then a maximum point was missing. After further analysis of the data between the two minimum points and estimation of the value of $\mathrm{d} P$, the missing maximum point was found out;

(c) When $\mathrm{d} W 2$ was within the normal range, if two minimum points were identified between two maximum points and this $d W 1$ was below the lower limit, then a minimum point was detected false. Comparing the amplitudes of these two minimum points, and the smaller one was chosen as the final minimum point, under the premise that $\mathrm{d} T$ was within the normal range;

(d) When $d W 2$ was within the normal range, if no maximum point was identified between two minimum points and $\mathrm{d} W 1$ was out of the normal range, then a minimum point was missing. After further analysis of the data between the two maximum points and estimation of the value of $\mathrm{d} T$, the missing minimum point was found out.
Step (5) and (6) should be repeated till no more undetected or falsely detected points could be identified.

The flow chart of the algorithm is shown in Figure 3 and the discrimination rules in Figure 4. In the figure, $L_{1 \text { low }}=L_{2 \text { low }}=500 \mathrm{~ms}, L_{1 \text { high }}=L_{2 \mathrm{high}}=1200 \mathrm{~ms}$.

\section{Results and discussion}

Continuous ICP monitoring is usually applied to the patients with head injury, cerebral hemorrhage, cerebral tumor, etc. The continuous ICP signals in this study were monitored using Codman intraparenchymal microsensors (Codman and Schurtleff, Raynaud, MA) situated in the right frontal lobe. The ICP signals were recorded from nine patients, including three traumatic brain injury patients, three cerebral hemorrhage patients, two hydrocephalus patients, and one cerebral tumor patient. The sampling frequency is $400 \mathrm{~Hz}$. At the same time, electrocardiograph(ECG) and arterial blood pressure (ABP) signals were also recorded. Figure 5 shows 6-s simultaneously recorded ECG and ICP signals of a patient. It demonstrates that the ICP wave is related to the cardiac beat and is disturbed by noise, which makes it technically challenging to extract the single ICP wave because its feature points cannot be located accurately. Wavelet transformation as an effective de-noising method was applied to the continuous ICP wave. Figure 6 illustrates the decomposition results with the first generation wavelet and Figure 7 with lifting wavelet. By comparing the two figures, it could be seen that more noises existed in the detail signals of the first generation wavelet transform, which would affect the subsequent computation of modulus maxima if noises were severe. Thereby, the feature points of a single ICP wave could not be located accurately. In that case, artificial estimation was needed to obtain better de-noised results. However, the problem did not exist in the detail signals of Figure 7, thus it was simpler to de-noise the results with lifting wavelet.

The maximum and minimum values of a single ICP wave could be computed by employing modulus maximum algorithm to the de-noised continuous ICP wave [12]. Figure 8 illustrates that every single ICP wave is located precisely and identified effectively.

The parameters of every single ICP wave could be obtained by computing the eight ICP waves in 6-s time window shown in Figure 8, then the continuous ICP monitoring wave could be described with more parameters, which made the monitoring ICP data reflect the change of ICP more objectively and accurately.

Furthermore, to testify the validity of the algorithm developed in this article, clinical continuous ICP signals with the length of $30 \mathrm{~min}$ of nine patients are chosen. Based on our algorithm, the analyzed ICP signals were divided into $N$ segment/min first, here $N=2$, so every 


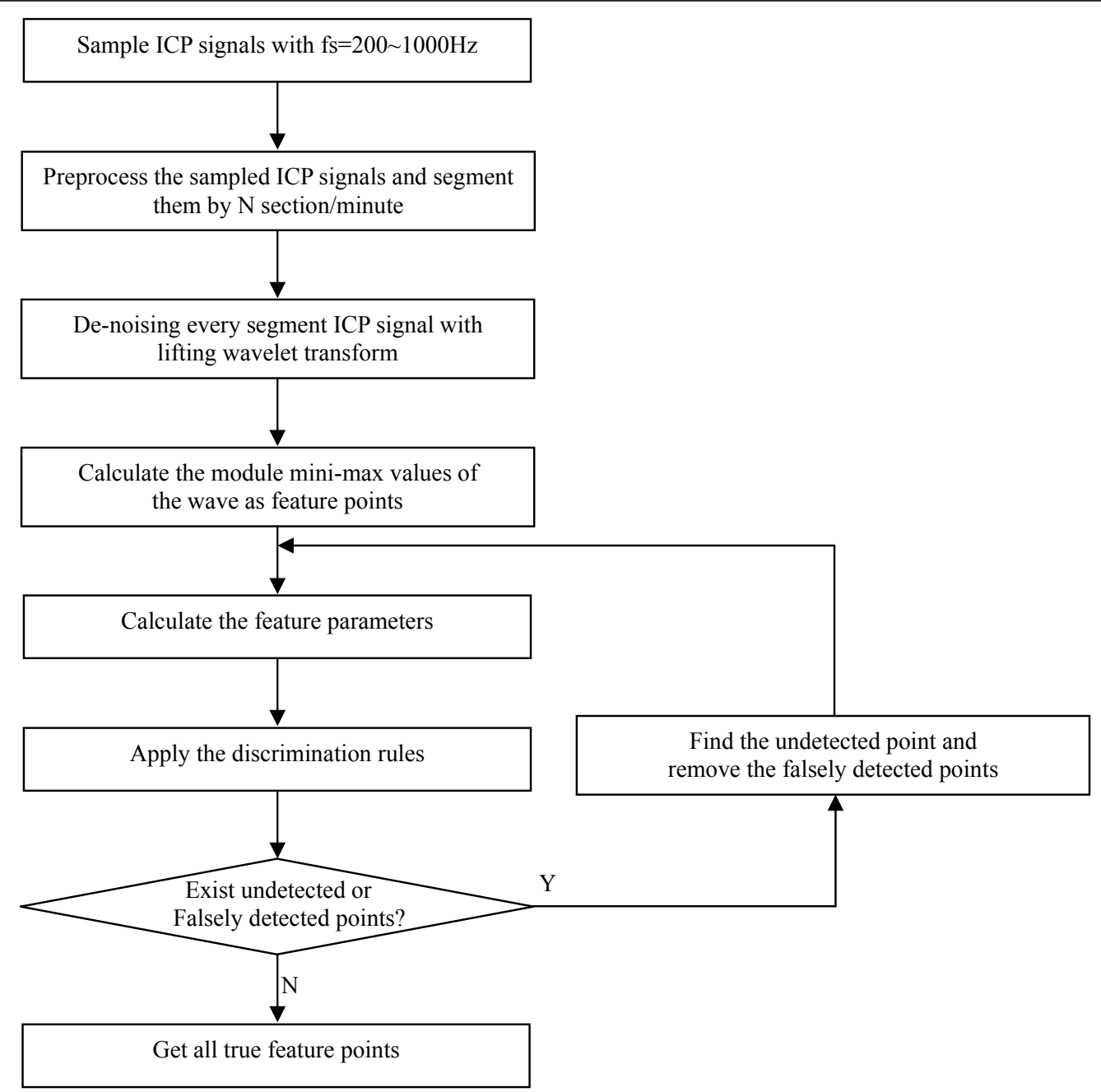

Figure 3 Flow chart of our algorithm.

ICP signal was divided into 60 segments with the length of $30 \mathrm{~s}$. Applying our algorithm to every segment ICP signals, Figure 9 shows the identification of a segment before using discrimination rules, and Table 1 shows the detected extreme points. The time duration between two adjacent sampling points was $2.5 \mathrm{~ms}$. As inferred from the table, the abnormal cases demonstrated in Section 4 occurred. By employing the algorithms, the false and missing extreme points could be detected, and further processing could remove the false extreme points and supplement the missing extreme points, as shown in Figures 9 and 10. In Figure 9, a green square represents that multi-maximum points exist between two minimum points; a megenta square represents that multi-minimum points exist between two maximum points; a black pentagram represents that a maximum point is missing nearby; a blue diamond represents that a minimum point is missing nearby. In Figure 10, a green square represents the reconfirmed maximum point in the false ones; a black square represents the missing maximum point; a megenata square represents the reconfirmed minimum point in the false ones; a blue diamond represents the missing minimum point. Use the discrimination rules repeatedly, till no more undetected and falsely detected extreme points can be discriminated.

For all the continuous ICP waves of nine patients, compared with the diagnosis results of clinical expertise, the analysis results with our algorithm are shown in Table 2. It can be seen that the accuracy rate was improved from 92.95 to $98.58 \%$ by using our algorithm with discrimination rules after lifting wavelet. Therefore, the method described in this article has given a possibility for the clinical use of ICP waveform.

\section{Conclusions}

A new novel method was developed to identify single ICP wave based on lifting scheme and the discrimination rules. In this way, not only mean ICP but also 


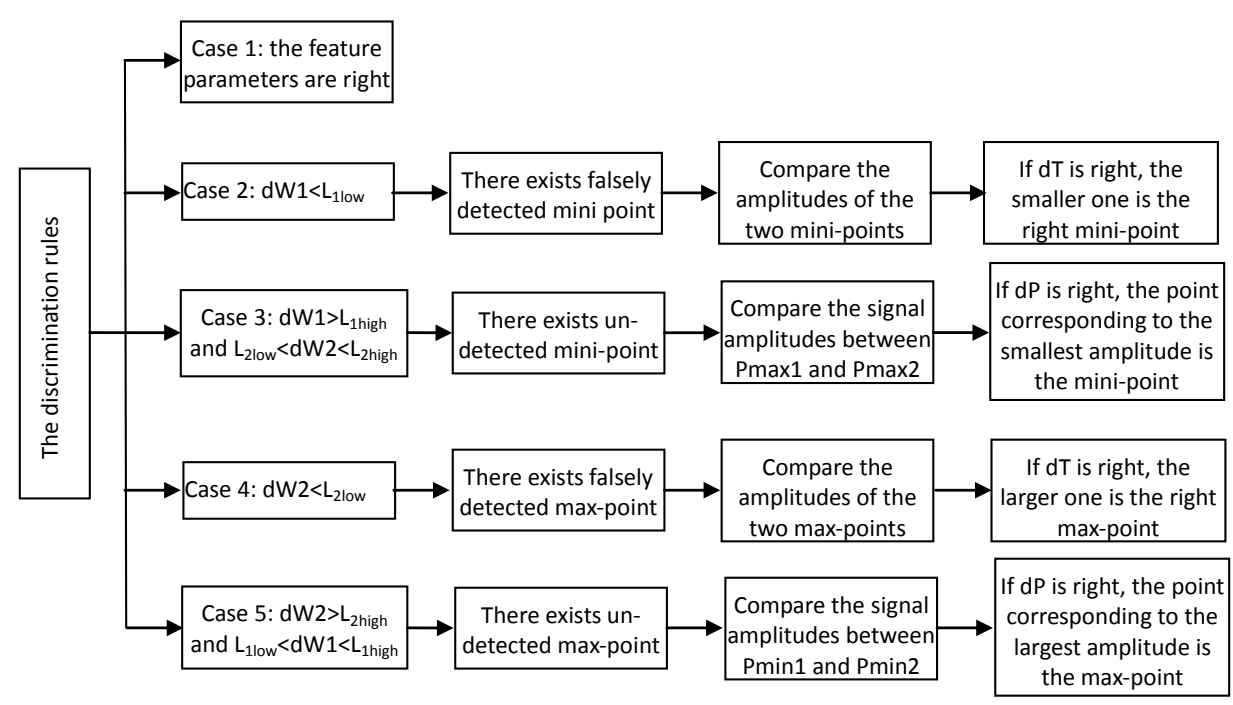

Figure 4 Discrimination rules.
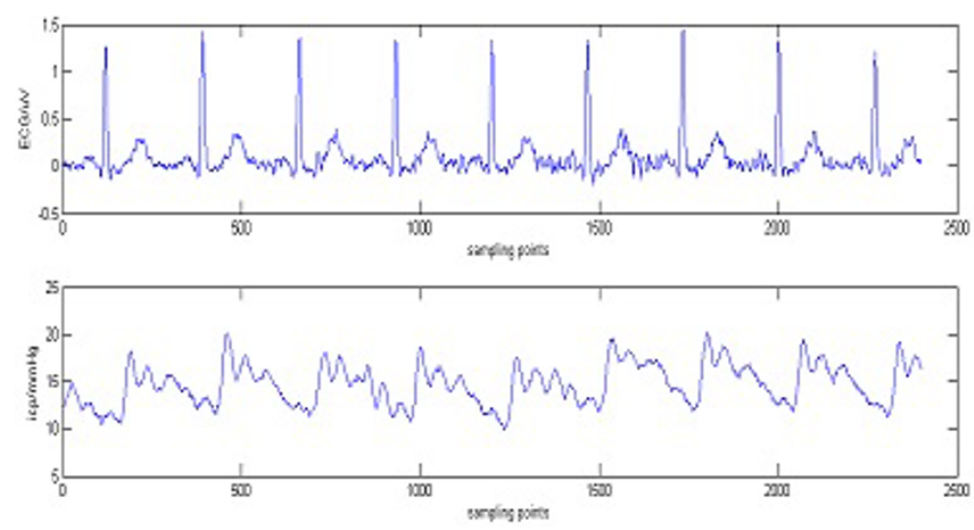

Figure 5 ECG and ICP signals.

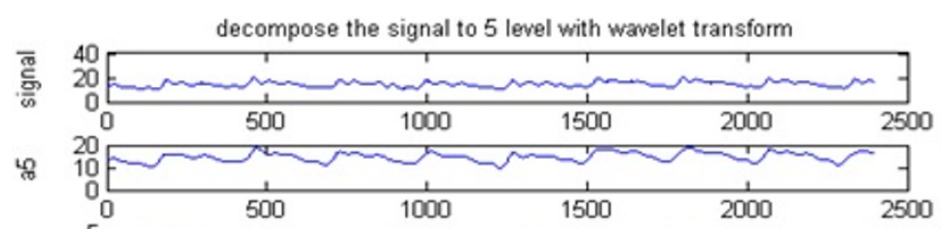

s8 ${ }_{-5}^{5}{ }_{0}^{5}$

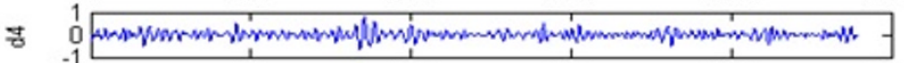

$\begin{array}{cccccc}-1 & 1 & 1 & 1 & \\ 0 & 500 & 1000 & 1500 & 2000 & 2500\end{array}$

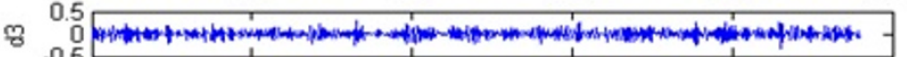

$\begin{array}{cccccc}-0.5 & 50 & 1000 & 1500 & 2000 & 2500\end{array}$

ช ${ }_{0.5}^{0.5}$
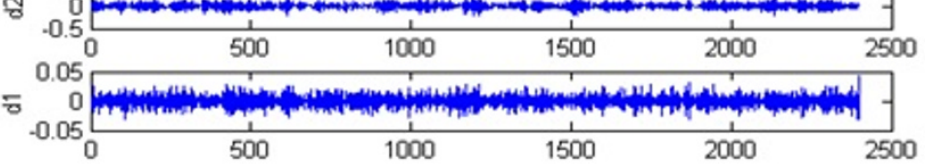

Figure 6 Continuous ICP signal decomposed with the first generation wavelet. 


Figure 7 Continuous ICP signal decomposed with lifting wavelet.
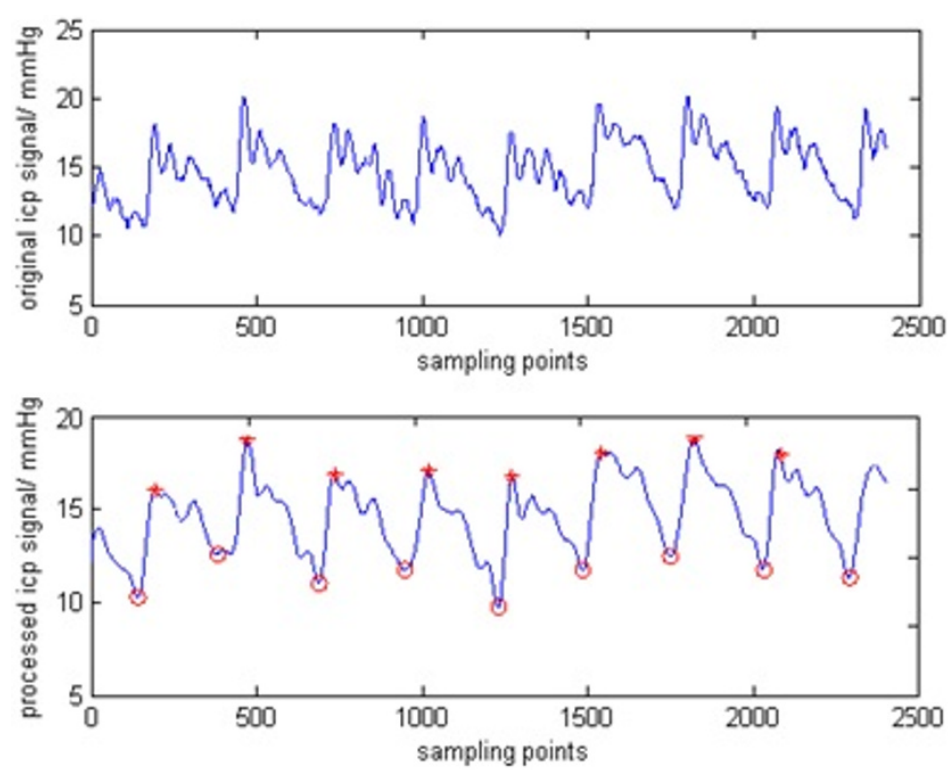

Figure 8 Identification of single ICP waves during 6-s time window.

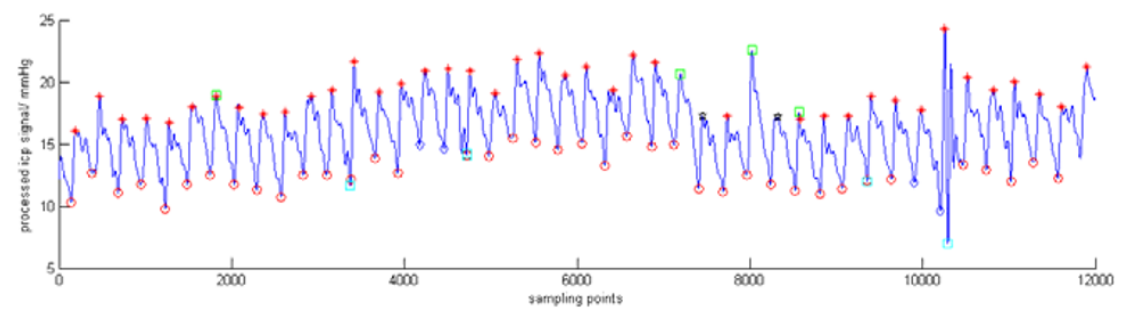

Figure 9 Detected extreme points with lifting wavelet and abnormal cases found out by discrimination rules. 


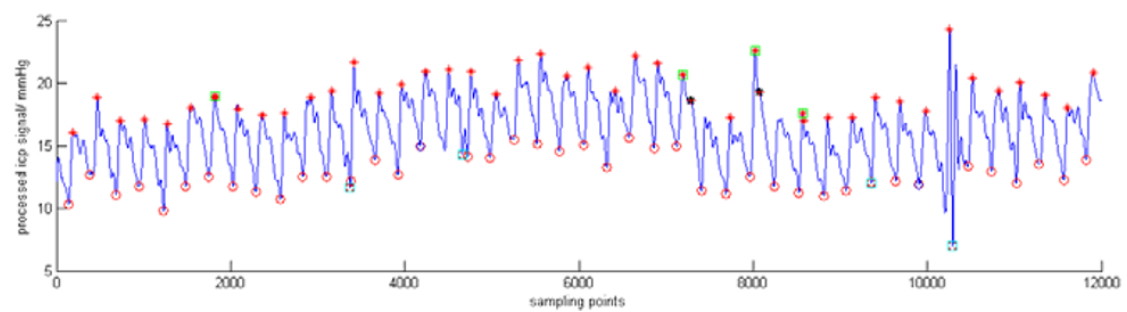

Figure 10 Detected and determined extreme points with our algorithm.

Table 2 Analysis results with our algorithm

\begin{tabular}{|c|c|c|c|c|c|c|}
\hline \multirow[t]{2}{*}{ Patients } & \multicolumn{3}{|c|}{ Before using discrimination rules } & \multicolumn{3}{|c|}{ After using discrimination rules } \\
\hline & Undetected & False detected & Detected extreme points & Undetected & False detected & Detected extreme points \\
\hline 1 & 60 & 140 & 2560 & 25 & 18 & 2717 \\
\hline 2 & 58 & 143 & 2558 & 24 & 18 & 2717 \\
\hline 3 & 62 & 138 & 2560 & 20 & 20 & 2720 \\
\hline 4 & 55 & 140 & 2565 & 18 & 16 & 2726 \\
\hline 5 & 58 & 140 & 2560 & 22 & 18 & 2718 \\
\hline 6 & 44 & 128 & 2478 & 21 & 18 & 2611 \\
\hline 7 & 52 & 130 & 2518 & 19 & 16 & 2665 \\
\hline 8 & 60 & 135 & 2565 & 22 & 16 & 2722 \\
\hline 9 & 59 & 138 & 2563 & 20 & 18 & 2722 \\
\hline Total & 508 & 1232 & 22927 & 191 & 158 & 24318 \\
\hline Accuracy rate & & & $92.5 \%$ & Accuracy rate & & $98.58 \%$ \\
\hline
\end{tabular}

single ICP wave amplitude and latency could be computed accurately; therefore, more information about ICP change could be provided in clinical practice.

\section{List of abbreviations}

ICP: intracranial pressure; ECG: electrocardiograph; ABP: arterial blood pressure.

\section{Acknowledgements}

The present work is supported by Scientific Research Foundation for Returned Researchers of Ministry of Education (Foreign Secretary Education, No. 1341), the Key Sci \& Tech Research Project of Chongqing (CSTC2009AB5200, CSTC2009AA5045, CSTC2010AA5049, CSTC2010AA5050) and Natural Science Foundation of Chongqing (CSTC2009BB5035). The author would like to thank Dr. Gurinder K Singh for critically reviewing the manuscript.

\section{Competing interests}

The authors declare that they have no competing interests.

Received: 19 April 2011 Accepted: 17 August 2011

Published: 17 August 2011

\section{References}

1. PK Eide, A new method for processing of continuous intracranial pressure signals. Med Eng Phys. 28, 579-587 (2006). doi:10.1016/j. medengphy.2005.09.008

2. CJ Kirkness, PH Mitchell, RL Burr, KS March, DW Newell, Intracranial pressure waveform analysis: clinical and research implications. J Neurosci Nurs. 32(5), 271-277 (2000). doi:10.1097/01376517-200010000-00007

3. PK Eide, Intracranial pressure parameters in idiopathic normal pressure hydrocephalus patients treated with ventrilculo-peritoneal shunts. Acta Neurochir (Wien). 148, 21-29 (2006). doi:10.1007/s00701-005-0654-8
4. X Hu, P Xu, S Asgari, P Vespa, M Bergsneider, Forecasting ICP elevation based on prescient changes of intracranial pressure waveform morphology. IEEE Trans Biomed Eng. 57(5), 1070-1078 (2010)

5. F Scalzo, P Xu, S Asgari, M Bergsneider, H Xiao, Regression analysis for peak designation in pulsatile pressure signals. Med Biol Eng Comput. 47, 967-977 (2009). doi:10.1007/s11517-009-0505-5

6. M Balestreri, M Czosnyka, LA Steiner, E Schmidt, P Smielewski, B Matta, JD Pickard, Intracranial hypertension: what additional information can be derived from ICP waveform after head injury? Acta Neurochir (Wien) 146, 131-141 (2004). doi:10.1007/s00701-003-0187-y

7. X Hu, P Xu, F Scalzo, P Vespa, M Bergsneider, Morphological clustering and analysis of continuous intracranial pressure. IEEE Trans Biomed Eng. 56(3), 696-705 (2009)

8. J-H Zhang, K Janschek, JF Bohme, Y-J Zeng, Multi-resolution dyadic wavelet denoising approach for extraction of visual evoked potentials in the brain. IEE Proc-Vis Image Signal Process. 151(3), 180-186 (2004). doi:10.1049/ipvis:20040315

9. Z Z Ji, T Jin, S-R Qin, Signal feature extraction based upon independent component analysis and wavelet transform. Chin J Mech Eng. 18(1) 123-126 (2005). doi:10.3901/CJME.2005.01.123

10. W Sweldens, The lifting scheme: a custom-design construction of biorthogonal wavelet. Appl Comput Harmon Anal. 3(2), 186-200 (1996). doi:10.1006/acha.1996.0015

11. W Sweldens, The lifting scheme: a construction of second generation wavelets. SIAM J Math Anal. 29(2), 511-546 (1997)

12. W Wang, Y-T Zhang, G-Q Ren, Denoising by self-adaptive lifting algorithm based on modulus maximum analysis, in IEEE ICMTMA'09 Proceeding of the 2009 International Conference on Measuring Technology and Mechatronics Automation. 1, 449-452 (2009)

doi:10.1186/1687-6180-2011-43

Cite this article as: Ji et al:: Single wave extraction in continuous intracranial pressure signal with lifting wavelet transformation and discrimination rules. EURASIP Journal on Advances in Signal Processing 2011 2011:43. 